\title{
Visual Analysis of Dynamic Changes in Structured Data on the Basis of Colour Markers
}

\author{
A.A. Trubakova ${ }^{1}$, A.O. Trubakov² \\ Bryansk State Technical University, Bryansk, Russia \\ 1 ORCID: 0000-0003-0280-1760, trubakovaaa@gmail.com \\ 2 ORCID: 0000-0003-0058-1215, trubakovao@gmail.com
}

\begin{abstract}
One of the most important tasks for decision-making systems is obvious and intuitive visualization of input data. If they are displayed correctly and conveniently, this tool can become a very serious assistant for a decision-maker. Such a tool influences not only on the complexity of the decision-making process, but also the correctness and objectivity of the decisions made. Because of this so much attention is paid to this issue in various studies. However, nowadays the issue of displaying the dynamics of changes in structured data (data in which the observed value has an internal structure and consists of a large number of components) is not sufficiently developed. This paper focuses on these issues, provides a formal description of structured data, and suggests an approach to displaying the dynamics of their changes. The proposed approach to visualization allows to see both the general process of changing the observed value and the nature of changes in its internal structure. Separate charts give the opportunity to see the contribution of each component of a structured value and evaluate the dynamics of this contribution over time. The paper also shows the area of potential application of this approach, highlights its features and main prospects. Most of examples in the paper are based on the developed software package for modeling the situation with the spread of COVID-19 in Bryansk region, which used the proposed visualization option. There are also examples in which the proposed approach to visualization helps to get new information that is not visible when using other approaches to displaying structured data.
\end{abstract}

Keywords: Data Visualization, Structured Data, Making Decisions, SEIRD-model.

\section{Introduction}

It is an obvious fact that the amount of data that needs to be analyzed when making management decisions greatly grows every year. At the same time, a man remains responsible for the analysis and final decision, that is, they depend on the decision-maker (DM). Modern decision support systems (DSS), based on mathematical methods, can partially replace human resources at the stage of processing initial data, but not at the stage of final decision-making. The lack of clear and structured information during the decision-making process makes it difficult, due to processing data of various nature, frequency of information updates over a short period of time, while the support and decision-making is always left to DM. Due to this need for operational research, it is required to provide high-quality visualization of constantly growing and developing volume of data that rapidly appears as a result of global scientific research around the world [1]. Range of research that actively involve the visualization as a mean of interaction with any type of data is constantly expanding. In turn, this leads of course to a significant increase in data volume, complexity of their structure, the use in the analysis of new data types as auxiliary or even major, information sources [2]. 
It is especially important to develop visualization methods if we deal with analyzing unusual data, dynamically changing over time. In this case, visualization methods are faced with a very important and not easy task of visual and intuitive display of unrolling a certain value change over time, showing the nature of this change, analysis and cooperation with the accepted solutions, the result of these solutions. To do this, visualization types such as graphs, bar charts, and histograms are used.

However, the situation with visualization can become very complicated if the quantity is multicriteria [3] or consists of several components. In certain cases, it is useful and very important for DM to analyze not just the change in the observed value, but also the impact of measures taken on the structure of its components, the dynamics of changes in the structure and the contribution of components. This article is devoted to the visualization of such data.

\section{Visual analysis of the dynamics of changes in structured values}

\subsection{The concept of a structured value}

To begin with, we introduce a formalized concept of a structured value. This paper will consider the issues of visualizing the dynamics of changes in such data.

Let us introduce the concept of a structured value, the visualization of dynamic changes in which we will consider in this paper. Let's suppose we have some observed value $P$. This value includes several components $k_{i}$ :

$$
P=\sum_{i} k_{i}
$$

Thus, the observed value $P$ is decomposed into a number of components. And from the point of view of visualizing such values, two cases can be distinguished:

- $P$ value is decomposed into a small number of components (less than 5 );

- $P$ value consists of a large number of components.

Both of these cases are identical in terms of mathematical description, but have significant differences in visualization. If a small number of components a person is able to visualize and see their structure, then in the case of a large number visual representation becomes more difficult and visual analysis becomes more complex. Let us call the value described above structured. In this case, the dynamics of changes in the structured value will be represented by such a dependence, in which at each specific moment $t_{j}$ the value $P\left(t_{j}\right)$ will be defined as the sum of the components $k_{i}\left(t_{j}\right)$ :

$$
P\left(t_{j}\right)=\sum_{i} k_{i}\left(t_{j}\right)
$$

This paper is devoted to visualization principles of this dynamics and these values.

\subsection{Practical necessity of analysing the dynamics of changes in structured values}

Let's look at practical situations where visual analysis of structured data dynamics can be very useful.

During the quarantine period of the first half of 2020, a group of students and teachers at Bryansk State Technical University was engaged in constructing COVID-19 distribution models in AnyLogic. At the same time, a number of modeled and observed values were just composite and had the character described above.

One of the most important and critical indicators of spreading COVID-19 is the number of infected people (let's call it $I N F$ ). It is this indicator that primarily determines quarantine measures, the complexity of epidemic control in a particular region, and various management decisions $[4,5]$. To display and visually analyze the dynamics of changes in the number of cases over time, you can use the classic method of displaying the curve as a function graph.

However, as practice has shown, the total number of cases in a particular region (or even a country) does not adequately describe the situation. Even if this value is normalized by the 
region population (that is make the value relative), this is clearly not enough for the analysis. The fact is that $I N F$ value itself is a composite one by its nature and its constituent parts have quite different degrees of criticality and importance. For example, INF value can be decomposed into such components as the number of seriously ill patients (requiring connection to an artificial lung ventilator), the number of moderate-severity patients, and the number of patients who do not require hospital admission. Therefore, the same INF value in different regions may show the overall situation in completely different ways. To understand the situation correctly, it is important to see not only the indicator itself, but also the structure of its components, and evaluate the contribution of each component.

Besides, if the number of components is not very large, we can use traditional schemes for displaying and visual analysis, such as multiple graphs (for each criterion separately), column graphs, histograms and Temporal Networks. In recent years, the research community has also accumulated overwhelming evidence in favor of complex and heterogeneous connectivity patterns based on Temporal networks [6]. However, the situation becomes more complicated if the number of components increases. In this case, the visibility of traditional approaches is greatly reduced and attempts are being made to develop new methods and approaches in this way [7].

Let's take another example. According to the World Health Organization (WHO), the incidence and severity of the disease in COVID-19 depends very much on the age. Therefore, it can be very useful to analyze the number of cases (INF indicator introduced before), but only in the context of the age of cases. That is, the components of this indicator should be considered as the number of cases of what age contributed to the total number of cases. This indication and analysis can be useful for evaluating actions and decisions taken. For example, some restrictive measures may not lead to a decrease in the total number of cases, but may change the structure of this value, reducing the number of elderly patients, which is also a very important factor in the epidemic control [8].

At the same time, visual analysis by age is very time-consuming. It is difficult to estimate this indicator due to the large number of age options. Therefore, in most cases, reduction of the variants (components) is done by rounding the age to certain ranges [9]. An example of such rounding, taken from official statistics on age, gender, and disease, depending on the death of patients from infection caused by COVID-19 is shown in Table 1 . The data are based on an official document dated February 28, 2020 from the WHO-China Joint Mission report [10].

Table 1. WHO-China Joint Mission data of depending the disease on the age

\begin{tabular}{|l|l|l|l|}
\hline Age & $\begin{array}{l}\text { Number of } \\
\text { infected people ) }\end{array}$ & $\begin{array}{l}\text { Number } \\
\text { deaths }\end{array}$ & $\begin{array}{l}\text { of } \\
\text { The likelihood } \\
\text { of death from } \\
\text { COVID-19 }\end{array}$ \\
\hline 0-9 years & 416 & - & - \\
\hline 10-19 years & 549 & 7 & $0,2 \%$ \\
\hline 20-29 years & 3619 & 1 & $0,2 \%$ \\
\hline 30-39 years & 7600 & 18 & $0,2 \%$ \\
\hline 40-49 years & 8571 & 38 & $0,4 \%$ \\
\hline 50-59 years & 10008 & 130 & $1,3 \%$ \\
\hline 60-69 years & 8583 & 309 & $3,6 \%$ \\
\hline 70-79 years & 3918 & 312 & $8,0 \%$ \\
\hline 80+ years & 1408 & 208 & $14,8 \%$ \\
\hline
\end{tabular}

In the example given the age of patients is divided by 10 years. However, such rounding is associated with certain problems. Firstly, poorly chosen age ranges can lead to incorrect analysis and a strong distortion of the real situation. Secondly, by rounding up age ranges, we lose some of the information for analysis, which can be very significant. Thirdly, the selected ranges may vary greatly for different regions and countries, and to make the selection in each specific situation is a very time-consuming task [11]. Therefore, the important point is not to abandon the full range of ages, but to develop new principles for visualizing such data, which will take into consideration all the data in their original regardless of their gradation and components. 


\subsection{Existing approaches to displaying the dynamics of data changes}

There are a lot of ways to show the dynamics of data changes. Let us consider the most commonly used options by the example of dynamics of developing COVID-19 on the territory of Bryansk region. The graphs show the dynamics of infections, recoveries, deaths due to COVID-19, the dynamic number of new infections, identified on the territory of Bryansk region, compared with the previous day and according to days since the first revealed infected person.

The most common option for displaying these data is to use traditional charts. In this case, time is $\mathrm{X}$-axis, and the value under consideration is Y-axis (see Fig.1).

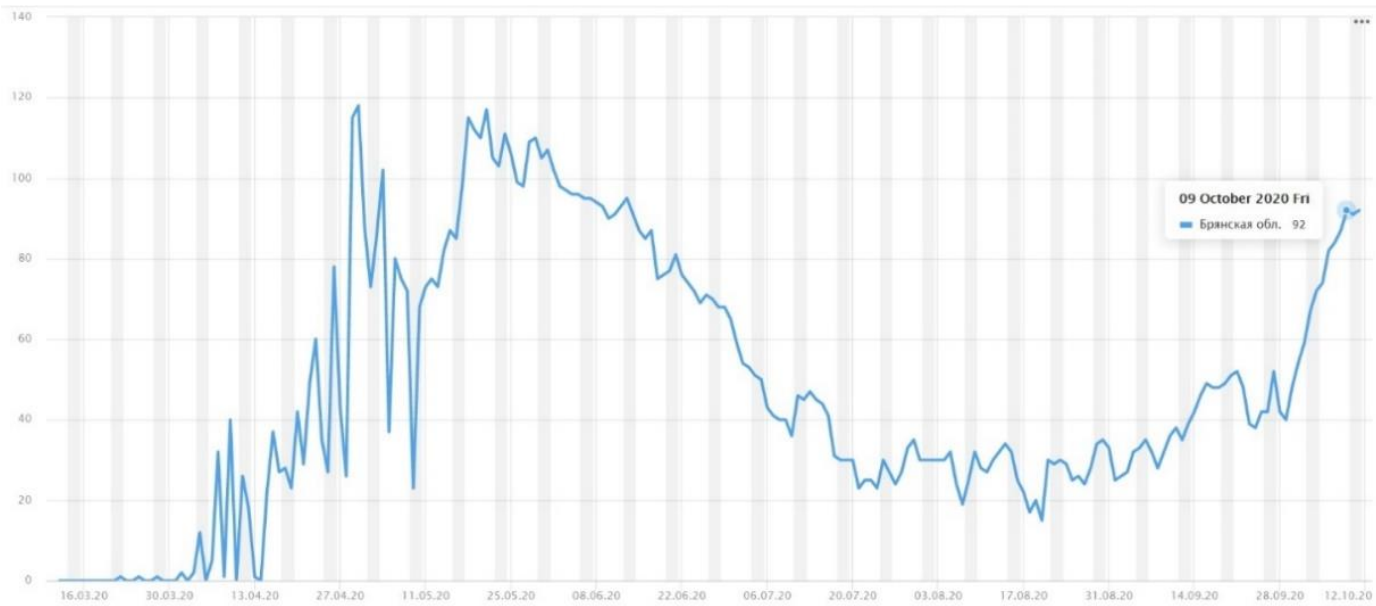

Fig. 1. The graph of changes in the number of cases of COVID-19 per day

If the data are structured, then several graphs are constructed for each of the components. An example of such graphs is shown in the first diagram of Fig. 2.

Число новых заражений, выздоровлений и смертей с начала марта Брянская область

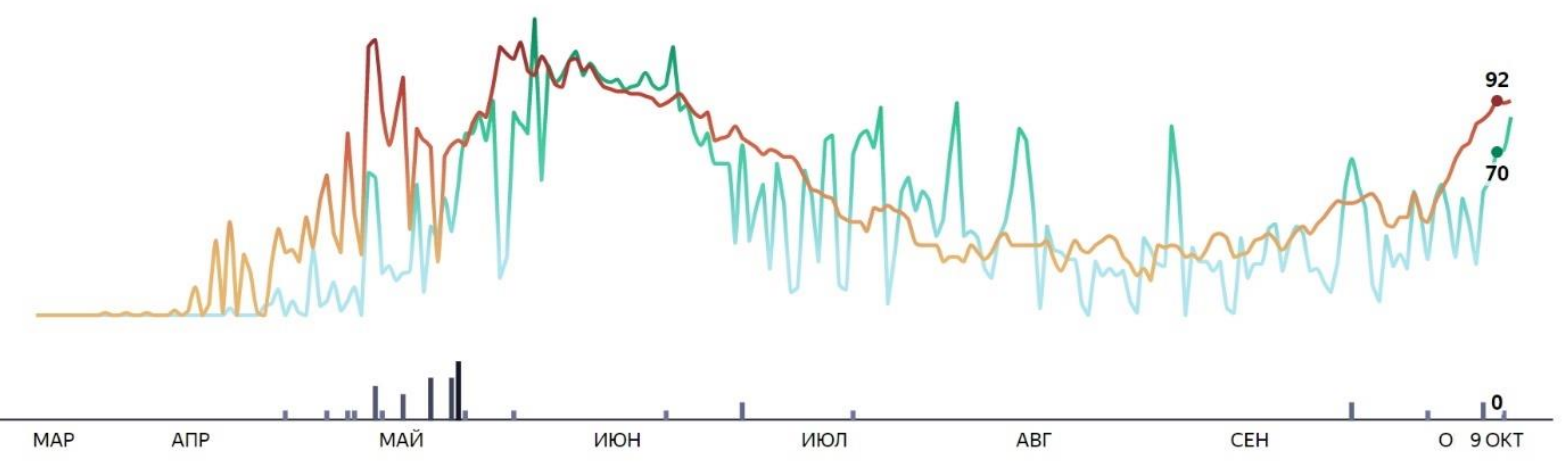

Fig. 2. The number of infected, recovered, dead people from COVID-19

However, it is good to analyze such graphs if the number of the observed values is small enough (one or two). If there are a lot of such values, the graph is difficult to read and it becomes very hard to draw any conclusions in this situation. Another problem is the difficulty of estimating the contribution of each component to the total. Based on such render methods, it is often impossible to analyze the overall situation of the epidemic growth in terms of the patient's age, gender, , concomitant diseases , activities, etc. [12].

Another option, which is also often found in open sources of statistics, is a column chart. In this case, each component is displayed as a separate column of its own color, and the dynamics is displayed as a set of such columns. According to Yandex, Apple and Otonomo data, there is given an example of a column chart grouping changes in the level of activity of the Russian population in the period from February to June (see Fig.3). 


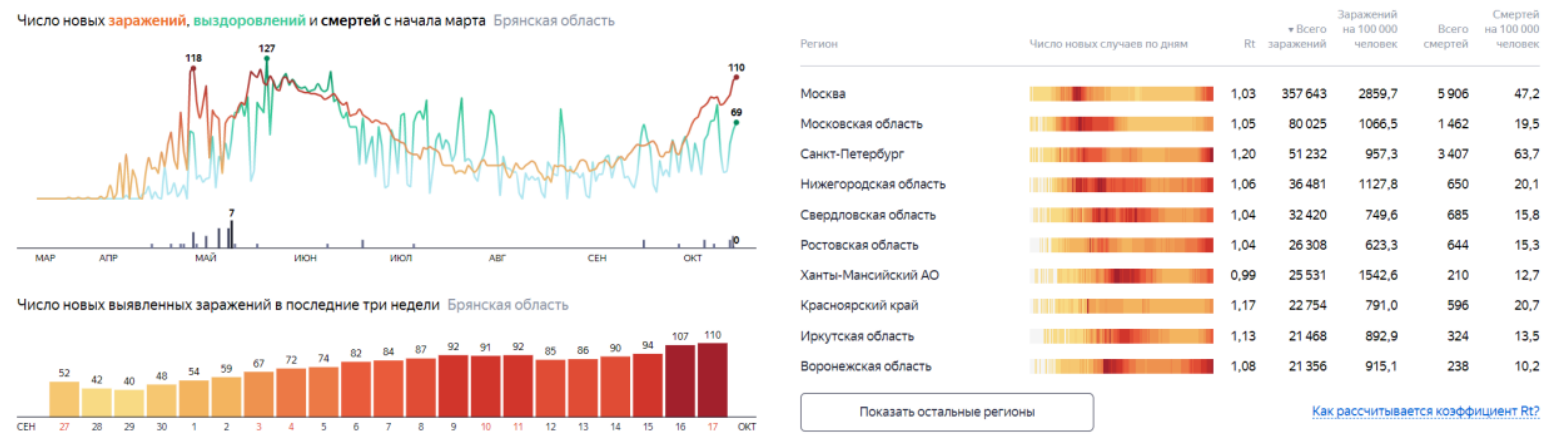

Fig. 3. Charts rendering the dynamics of COVID-19 in Bryansk region

Another fairly good rendering option is a radar chart and a pie chart [13]. In contrast to the previous versions, these methods are very good at visualizing the data structure, clearly showing the contribution of each component. However, they are very difficult to apply for showing the dynamics of changes in this data over time.

\section{COVID-19 spread modeling system and problems of vis- ualizing the dynamics of structured values in it}

Epidemic outbreaks in various regions represent a special situation with a high level of uncertainty. In some regions, there was a noticeable repetition of the situation in the same scenario. In others, the situation was significantly different. A software package in AnyLogic environment was developed in Bryansk State Technical University [14]. The authors suggested using several modeling directions. The mixed simulation model was based on a combination of approaches: discrete-event directed modeling, agent-based modeling, and the system dynamics section. The use of classical approaches to epidemic modeling based on SEIRD model was proposed as the main method [15]. SEIRD disease distribution model belongs to a class of so-called compartmental models, the essence of which is to divide the population into several groups, i.e.: $\mathrm{S}$ (susceptible) means the number of people susceptible to the disease, $\mathrm{E}$ (exposed) is a group of infected people who are in the incubative stage, I (infected) means infected, R (recovered) stands for the number of people who recovered after the infection, D (dead) is dead. Each of the specified parameters forms variables that are part of a system of differential equations, which can be used to predict the dynamics of the epidemic.

As a result of analyzing a series of experiments conducted with COVID-19 disease development model it is possible to visualize the rate of infection spread with different behavior of people and various parameters, conditions and constrains. The screenshot of the system is shown in Fig. 4. The system was presented at the contest for the best scientific work "Modern scientific achievements. Bryansk-2020".

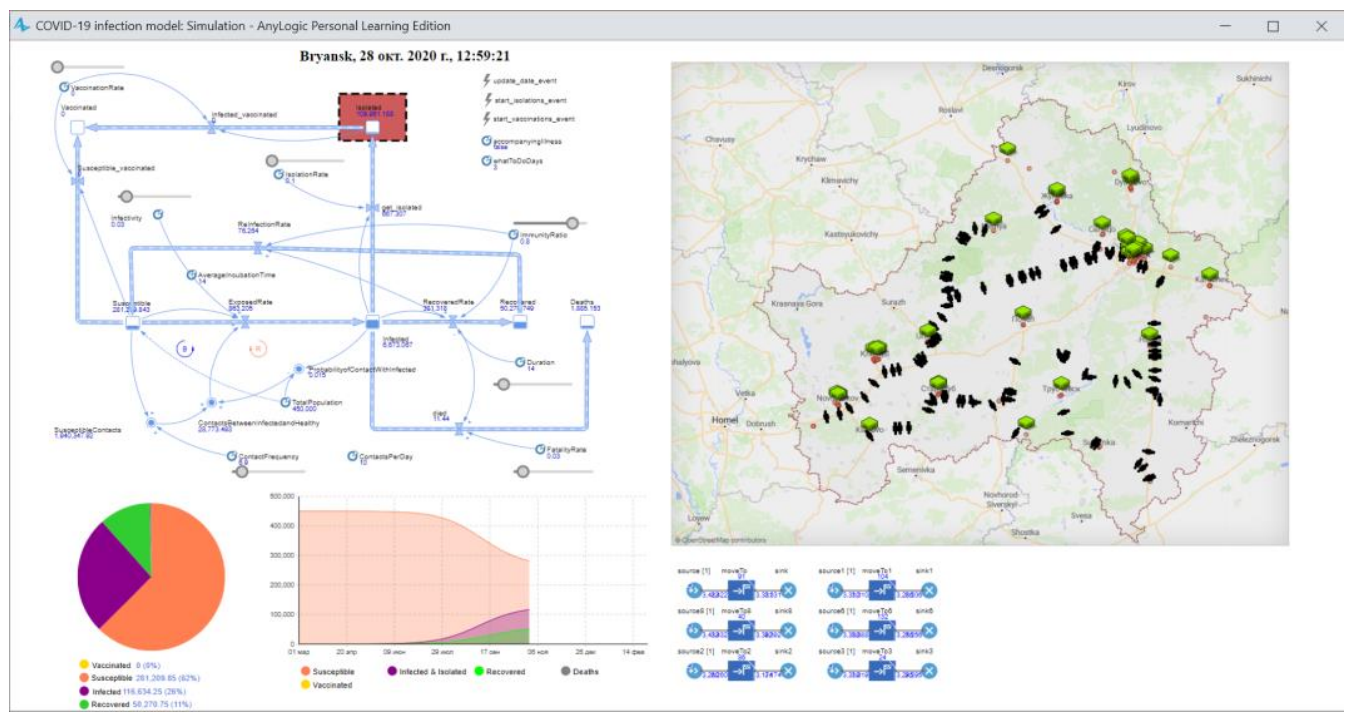

Fig. 4. System of modelling COVID-19 in Bryansk region 
Taking the known criteria as a basis, it is possible to demonstrate the development of infection spread, indicators of the level of disease severity [16]. We used a model that incorporated the main factors known at that time about the spread of infection among people. Specific indicators and coefficients were calculated based on similar data for Moscow region [17] and included in the complex for modeling the situation in Bryansk region. As a result, a model was built (see Fig. 5) that takes into account the following factors of COVID-19:

1. Immunity. The better the person's immunity, the lower is the probability of COVID-19 infection (ImmunityRatio parameter of double type, which takes value from o to 1). Each object Person in the constructor is assigned with a random value in the range specified above.

2. Social responsibility (IsolationRate parameter of double type has a default value of 0.45) [18]. According to the emergency response centre of the Russian Federation, the level of social responsibility is approximately $45 \%$ (exactly following the doctor's prescription and recommendations, as well as quarantine or self-isolation measures). The value of this parameter changes dynamically based on data received from Rospotrebnadzor (Russian Federal Service for Surveillance on Consumer Rights Protection and Human Wellbeing) and Johns Hopkins University.

3. ContactsPerDay parameter defines the average number of close contacts of the object Person per day.

4. The probability of contacts between people with different degrees of infection: the probability of contacts between infected people and healthy people (ProbabilityofContactWithInfected parameter), the frequency of contact, and maintaining social distance (ContactFrequency parameter).

5. Accompanying coronavirus diseases (factor of a person's going to the hospital in case of infection, accompanyingIllness parameter of boolean type).

6. The time to make a decision after infection symptoms onset is a factor that can be used to make a conclusion about the disease severity (whatToDoDays parameter is of int type and is set randomly from the above range for each object Person). According to statistics in the region [19], a person goes to the hospital or goes to self-isolation within 1-5 days. In this time range, a person begins to notice symptoms onset and deterioration of his health condition.

7. Mandatory self-isolation of people (start_isolations_event, if the threshold of sick people is exceeded, as well as based on data from the period of self-isolation in Bryansk region and orders of authorities - intervention).

8. The probability of virus spread is InfectionProbability parameter. The probability value depends on several factors, such as the use of personal protective equipment, wearing masks, washing hands, or touching the person's respiratory organs and mucosa.

Based on the tasks and factors that affect the development of the spread of Covid-19 infection, the model is built using a system of differential equations described below:

$$
\begin{gathered}
\text { ExposedRate }=\text { Susceptible } \cdot \text { ContactsBetweenInfectedandHealthy } \cdot \text { Infectivity } \\
\text { InfectionRate }=\frac{\text { Exposed }}{\text { AverageIncubationTime }} \\
\text { RecoveredRate }=\frac{\text { Infected }}{\text { Duration } \cdot \text { ImmunityRatio }} \\
\text { Died }=\text { RecoveredRate } \cdot \text { FatalityRate } \\
\text { ReInfectionRate }=\text { RecoveredRate } \cdot(1-\text { ImmunityRatio })
\end{gathered}
$$

where: ExposedRate is the number of sick people per unit of model time, Susceptible is the number of people susceptible to infection with Covid-19 (taking into account factors such as weakened immunity, being in public places of large crowds, etc.), ContactsBetweenInfectedandHealthy is the average number of contacts between infected and healthy people, Infectivity is the probability of infection during the spread of an epidemic, InfectionRate is the rate of the disease progression, AverageIncubationTime is the average value of the incubation period, RecoveredRate is the number of people who set recovered from an illness (the absence of a virus in the body during testing) per unit of model time, Infected - infected people, Duration - mean time of disease progression, FatalityRate - number of people who 
died per unit of model time, Died - percentage of mortality (probability of death based on patient statistics).

The structure of the simulation model of the disease development according to the unit of model time equal to one day is shown in Fig. 5 .

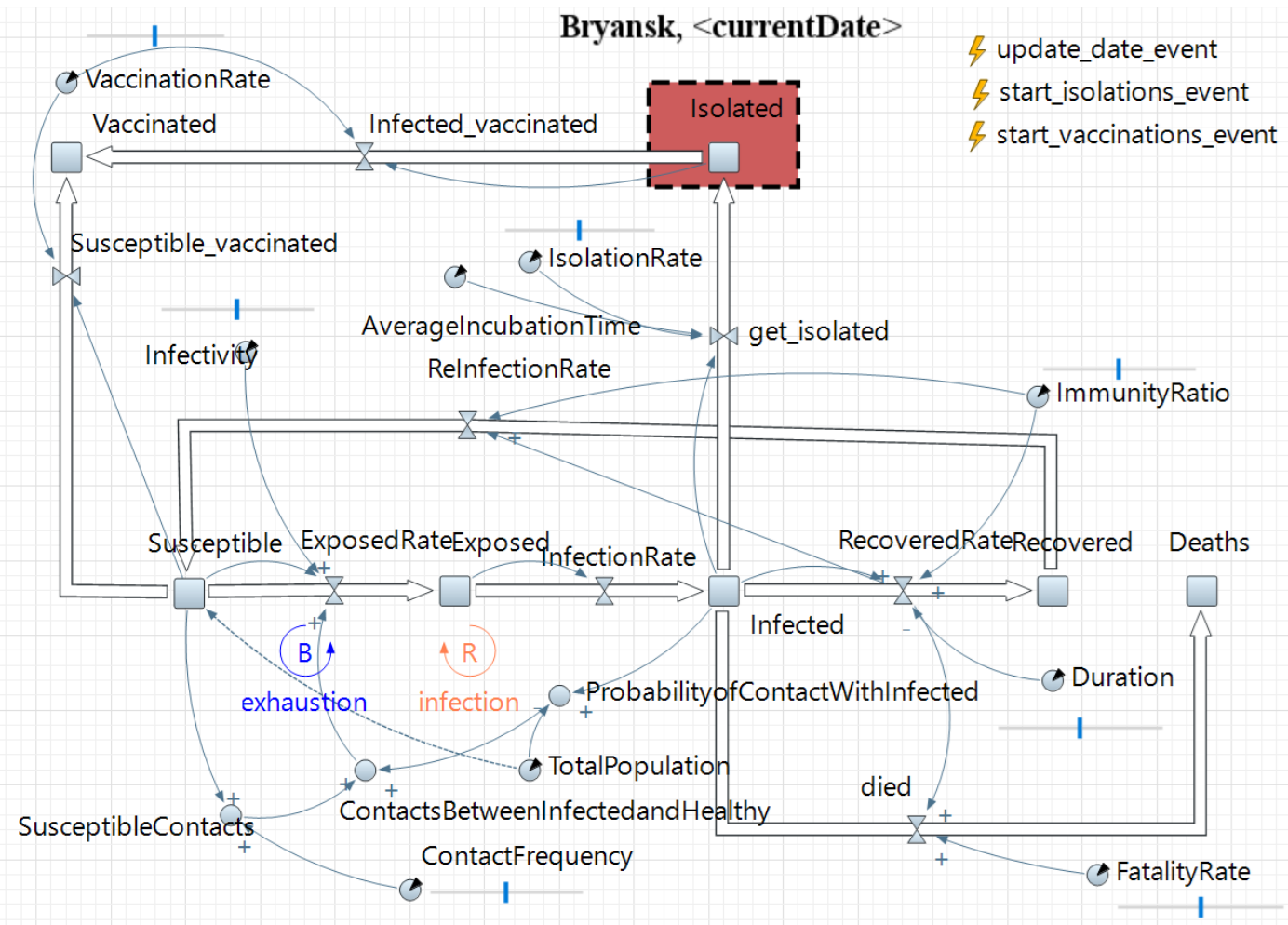

Fig. 5. System Dynamics Model of the spread Covid-19

The color marker approach described below was used to display and visualize data on the dynamics of changes in structured values. This made it possible to present the results more clearly.

The data on the infection spread in Bryansk region obtained during modeling on the basis of open and publicly available information shows the feasibility of the model. The discrepancy between the currently modeled and real curves does not exceed $8 \%$.

\section{The proposed solution for visualizing the dynamics of changes in structured values based on color markers}

\subsection{Using color markers to display the structure of a composite value}

As it was described above, in the framework of research and simulation of the situation with spreading COVID-19, conducted in Bryansk State Technical University, we used an approach to show the dynamics of changes in structured values, which is conventionally called the method of color stripes or color marker graph. The main idea of this approach is as follows. Let us assume that we have a certain value $P(t)$, which value changes over time (the value under study depends on t parameter). In this case, this value consists of different components. That means that at $t$ time moment $P(t)$ value can be decomposed into some components:

$$
P(t)=\sum_{i} k_{i}(t)
$$

For visual image of these data (both the graph of changes in the value itself and its components), we assign each component part $k_{j}$ a certain color (the so-called color marker):

$$
\left\{k_{1} \leftrightarrow \text { цвет }_{1} ; k_{2} \leftrightarrow \text { цвет }_{2} ; \ldots ; k_{n} \leftrightarrow \text { цвет }_{n}\right\} .
$$

Then the curve of the value change $P(t)$ is rendered as a standard graph, plotting time along the abscissa axis, and the observed value itself along the ordinate axis. At the same time, $k_{j}(t)$ 
component parts should be painted with the corresponding color marker. In this case, the graph of changes $P(t)$ will look like color bars of different widths (depending on the value of $k_{j}(t)$ components).

Now let us consider the example described in the previous chapter (analysis of changes in the number of cases in the context of disease severity). Let the analyzed value $P(t)$ be the total number of infected by COVID-19 at a certain time (INF value introduced above). At the same time, as it is described above, not the graph of changes in the number of cases is of interest, but the analysis of the components of this number, that is how many of these cases are severe, how many are moderate, how many people do not require hospital admission, and how many are asymptomatic. In this case this value at time moment ti is decomposed as follows:

$$
\operatorname{INF}(t)=\sum_{i=1}^{4} k_{i}(t) \text {. }
$$

where: $k_{1}(t)$ is the number of seriously ill at time moment $t ; k_{2}(t)$ is the number of hospital moderate-severity patients; $k_{3}(t)$ is the number of infected people who do not need hospital admission; $k_{4}(t)$ is the number of asymptomatically infected.

Let us assign a color marker to each value. Then the general graph of changes in the epidemiological situation will take the form shown in Fig. 6.

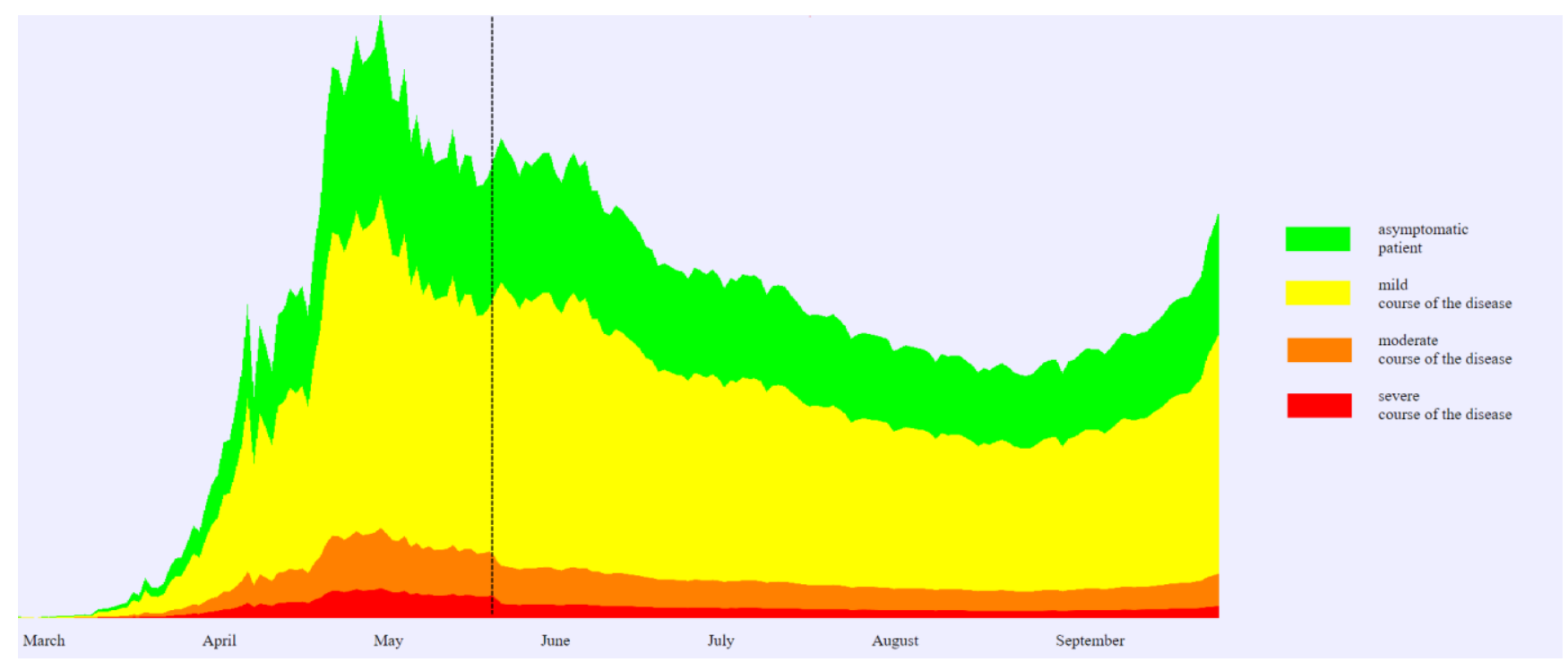

Fig. 6. Graph for showing a structured value in the form of color markers in the context of disease severity

This graph is a more visual way to show than tabular data or other options discussed earlier. This display provides more information for analysis and additional conclusions, allows to see patterns or effects that are not visible in other versions of the image.

A distinctive feature of displaying with color markers is that this method allows decision maker to perform visual analysis that was previously unavailable when displaying as a regular graph. For example, Fig. 6 shows one feature according to the graph given. At time moment $t_{k}$ marked on the graph with a stipple line, some measures were taken that did not significantly reduce the increase in the total number of patients and significantly changed, the spread of COVID-19 (INF value graph saves its nature). However, the decisions made at this moment allow to change the structure of $I N F$ value significantly in the direction of reducing the number of seriously ill $\left(k_{1}\right)$ and moderate-severity $\left(k_{2}\right)$ patients which is more important than general trends of the target indicator. Fig. 6 shows these changes and gives the opportunity of visual analysis and search of similar periods. For example, the exponent form of the curve on the graph of epidemic development under normal conditions. This form determines the rapid growth of morbidity rate, which in turn greatly increases the burden on health authorities, thereby increasing mortality due to the lack of proper medical care and appropriate equipment. When a person's social activity decreases by five times (self-isolation), there is a smooth evenning of the curve on the graph. This scenario increases the overall duration of the 
epidemic, but reduces the burden on health care, thereby reducing the number of patients who died. However, in the example given above (Fig. 6) there is a number of difficulties which make the visual analysis of the structure harder to realise. These difficulties include:

- $\quad$ in areas with a low INF target value, the color marker bars become narrow enough that any changes in them are not visible;

- $\quad$ in areas with a sharp change in the target value of INF, a jump in the target indicator makes it difficult to analyze visually $k_{i}$ components included in it.

In order to overcome these difficulties we suggest switching from absolute numbers to relative ones, i.e. construct color bars in a normalized variation:

$$
\begin{gathered}
k_{i}^{\prime}(t)=\frac{k_{i}(t)}{\operatorname{INF}(t)}, \\
\sum_{i=1}^{n} k_{i}^{\prime}(t)=1 .
\end{gathered}
$$

In such variant the fluctuations of the target indicator or a small value will not make it difficult to perceive the structure. The analysis of changes in the structure becomes even clearer and any changes or external influences that displace the internal components of the value become clearer (see Fig. 7).

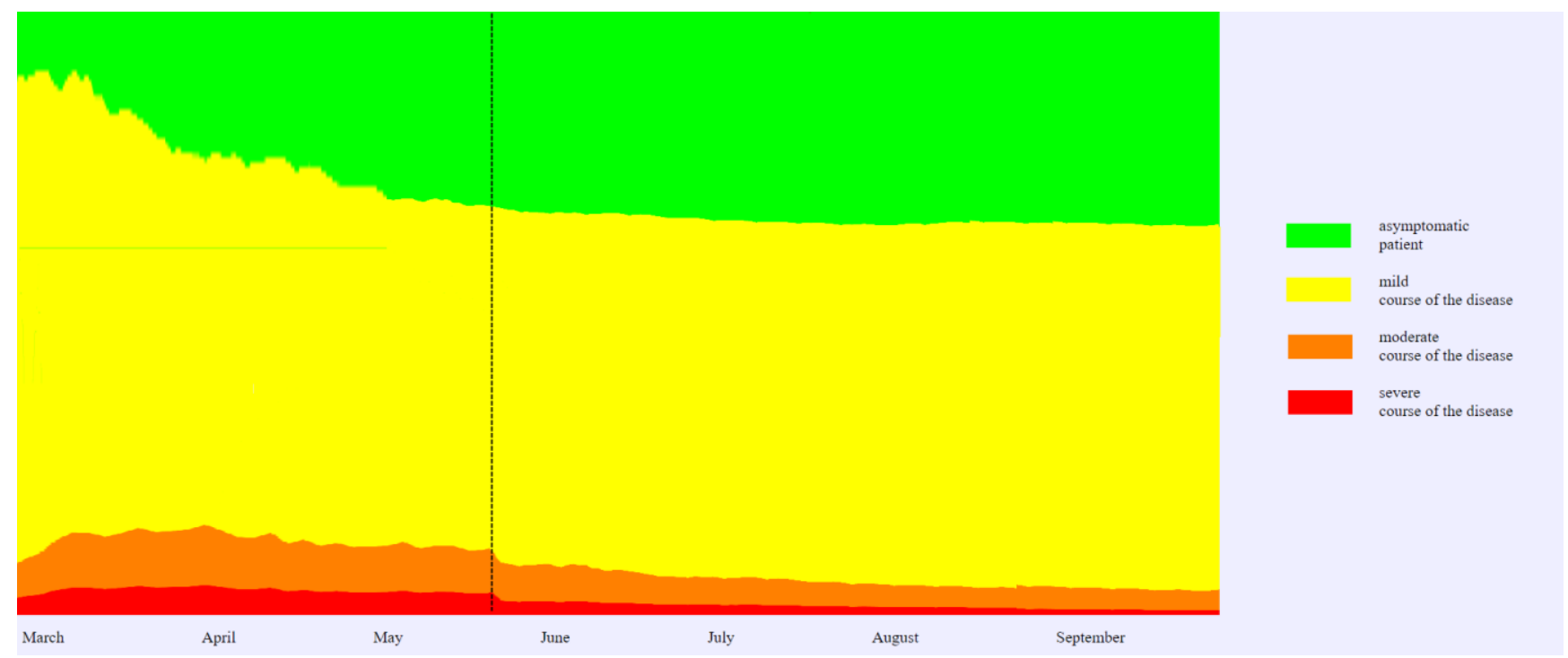

Fig. 7. Graph of structural changes

\subsection{Method generalisation for continuous data}

Let us consider another example of dynamic changes of structured data. In this case the observed value $P(t)$ consists of a large number of $k_{i}$ components. An example of such data is the previously considered analysis of morbidity rate in the context of patients' age. As practice has shown with the spread of COVID-19, this parameter is also very important for analyzing the situation and making management decisions.

Formally, this task is expressed as follows. As before, we will use the number of patients at time moment $t-I N F(t)$ as the target value. However, we will consider the value of the composite value $k_{\text {age }}(t)$ as the number of cases at time $t$ at the age, where age is in the range:

$$
0 \leq \text { age } \leq \text { MaxAge, }
$$

where MaxAge is the maximum age of infected people.

Visual representation of such a value by conventional methods (graphs, charts, tables) is quite problematic as the number of components is high (in the extreme case age can be a continuous value) [20,21].

Very often, to analyze and interpret such data, rounding the parameter and switching to certain ranges are used. For example, the all-Russian operational headquarters for publications it was offered to replace age parameter with the following ranges:

- children, teenagers, and young people (under 30 years old); 
- middle-aged people (30-49 years old);

- people aged 50-59 years;

- old people over 60 years old.

However, this division is very conditional. This is confirmed by numerous publications in which the age ranges may be completely different. This is due to the fact that it is quite difficult to identify the most objective ranges for analysis. First of all, the following issues cause difficulties:

- the need for preliminary data analysis and the introduction of additional criteria for forming ranges;

- the potential for changing ranges over time, which leads to unreliability of data if hard boundaries are introduced;

- loss of information content and part of the information due to rounding data and bringing them to predefined ranges;

- increase of time required for data analysis due to the need for an additional step (selection of range boundaries);

- difficulties in arranging border ranges that depend on external influences (for example, range boundaries may have geographical or other dependencies).

In addition, it is worth noting that the selection of pre-defined ranges and rounding the observed value automatically lead the problem to analysis in purely subjective values, and objectivity of the display results will strongly depend on how well or unsuccessfully the range boundaries were selected.

To analyze these data, we offer a slightly different approach. Due to the difficulties in selecting ranges, we suggest abandoning them and visualizing the data not as color bands, but as a continuous gradient. To do this, a smooth color change is compared to the age and the target value graph is filled in according to the obtained values of the composite components. The resulting graph is shown in Fig. 8.

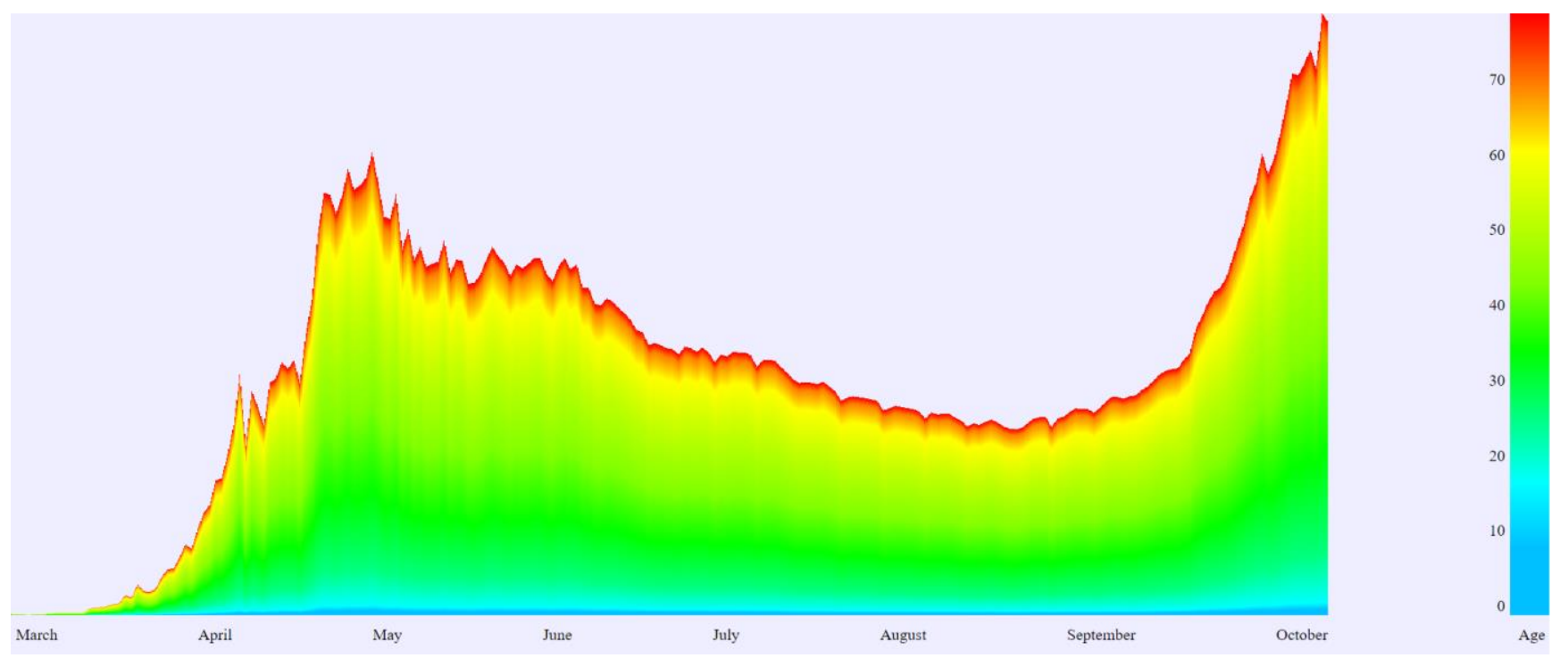

Fig. 8. Graph of showing structured value by means of color markers in the context of patients' age

This display does not require analysis and selection of rounding limits. Data can be visualised immediately until they are not processed. In this case, the composition patterns will automatically adjust and display adequately, regardless of any external or internal influences.

\section{Conclusion}

In this paper, we have proposed a method that combines analysis and visualization of structured data. Most of the examples in the paper are given on the situation with COVID-19 development. This is due to the fact that we have tried and tested this visualization approach on the basis of the corresponding complex. However, the proposed method of displaying the dy- 
namics of changes in the data structure in the form of color markers and gradients can be used in absolutely any areas where there is a need to display the dynamics of composite values, see and analyze the structure of these values.

\section{References}

1. Podvesovskii A.G., Isaev R.A.: Constructing Optimal Visualization Metaphor of Fuzzy Cognitive Maps on the Basis of Formalized Cognitive Clarity Criteria // Scientific Visualization, 2019, Vol. 11, Num. 4, P. 115-129. - DOI: 10.26583/sv.11.4.10

2. Zakharova A., Shklyar A. Basic principles of data visual models construction, by the example of interactive systems for 3D visualization // Scientific Visualization, 2014, Vol. 6, Num. 2, P. 62-73.

3. Bondarev A.E., Galaktionov V.A.: Generalized Computational Experiment and Visual Analysis of Multidimensional Data // Scientific Visualization, 2019, Vol. 11, Num. 4, P. 102-114. - DOI: 10.26583/sv.11.4.09

4. Russell Timothy W., Hellewell J., Jarvis Christopher I., van Zandvoort K., Abbott S., Ratnayake R., Flasche S., Eggo R.M., Edmunds W.J., Kucharski A.J. Estimating the infection and case fatality ratio for coronavirus disease (COVID-19) using age-adjusted data from the outbreak on the Diamond Princess cruise ship, 2020, https://doi.org/10.2807/1560-7917.ES.2020.25.12.2000256, last accessed 2020/07/10.

5. Yang L.Y., Yan L.M., Wan L., Xiang T.-X., Le A., Liu J.M., Peiris M., Poon L.L.M., Zhang W. Viral dynamics in mild and severe cases of Covid 19. Lancet Infect Dis, 2020.

6. Kapoor A., Ben X., Liu L., et al. Examining COVID-19 Forecasting using Spatio-Temporal Graph Neural Networks, 2020.

7. Zakharova A.A., Korostelyov D.A., Fedonin O.N.: Visualization Algorithms for Multicriteria Alternatives Filtering // Scientific Visualization, 2019, Vol. 11, Num. 4, P. 66-80. - DOI: 10.26583/sv.11.4.06

8. Murray C.J.L. Forecasting COVID 19 impact on hospital bed-days, ICU-days, ventilatordays and deaths by US state in the next 4 months. IHME COVID 19 health service utilization forecasting team, https://doi.org/10.1101/2020.03.27.20043752, last accessed 2020/07/10.

9. Khrapov P.V., Loginova A.A. Mathematical modelling of the dynamics of the coronavirus COVID-19 epidemic development in China // International Journal of Open Information Technologies, 2020, $\quad$ Vol. $\quad 8(4), \quad$ P. http://www.injoit.org/index.php/j1/article/view/908/874, last accessed 2020/07/10.

10. Matveev A.V. Mathematical modeling of evaluating the effectiveness of measures against the spread of COVID-19. // Natsionalnaya Bezopasnost I Strategicheskoe Planirovanie (National Security and Strategic Planning), 2020, Vol. 1(29), P. 23-39.

11. Kosara R. Presentation-oriented visualization techniques // IEEE Comput Grap Appl, 2016, Vol. 36, P. 80-85.

12. Koltsova E.M., Kurkina E.S., Vasetsky A.M. Mathematical modeling of the spread of COVID-19 coronovirus epidemic in a number of European, Asian countries, Israel and Russia // Economic problems and legal practice, 2020, Vol. 2

13. Wang D.Q., Guo D.H., Zhang H. Spatial temporal data visualization in emergency management: a view from data-driven decision // Proceedings of the 3rd ACM SIGSPATIAL Workshop on Emergency Management, 2017, P. 1-7.

14. AnyLogic, https://www.anylogic.ru/, last accessed 2020/10/15.

15. Jüni P., Rothenbühler M., Bobos P., Thorpe K.E., da Costa B., Fisman D., Slutsky A.S., Gesink D. Impact of climate and public health interventions on the COVID-19 pandemic: A prospective cohort study. CMAJ May 08, 2020.

16. Yang X, Yu Y, Xu J, et al. Clinical course and outcomes of critically ill patients with SARSCoV-2 pneumonia in Wuhan, China: a single-centered, retrospective, observational study. Lancet Respir Med, 2020, Vol. 8(5), P. 475-481.

17. Borisov V.V., Kruglov V.V., Fedulov A.S. Fuzzy models and networks. - M.: Hot line Telecom, 2012, P. 284. 
18. Rodkin M.V., Shikhova N.M. Mathematical modeling of COVID-19 epidemic, an attempt to forecast // Uralian Geological Journal, 2020, Vol. 3.

19. World Health Organization. Coronavirus disease 2019 (COVID-19): Situation Report - 38 from 27 February 2020, http://www.who.int/docs/default-source/coronaviruse/ situation-reports/20200227-sitrep-38-covid-19. pdf, last accessed 2020/o7/10.

20.Dimara E., Perin C. What is interaction for data visualization? // IEEE Trans Visual Comput Graph, 2020, Vol. 26, P. 119-129.

21. Robertson G, Fernandez R, Fisher D, et al. Effectiveness of animation in trend visualization // IEEE Trans Visual Comput Graph, 2008, Vol. 14, P. 1325-1332. 